\title{
Development of Mu Rhythm in Infants and Preschool Children
}

\author{
M. Berchiccia,b T. Zhang ${ }^{\text {e }} \quad$ L. Romero ${ }^{f} \quad$ A. Peters $^{f} \quad$ R. Annett ${ }^{g} \quad$ U. Teuscher ${ }^{f}$ \\ M. Bertollo ${ }^{a, b}$ Y. Okada ${ }^{\text {h }} \quad$ J. Stephen ${ }^{f}$ S. Comani ${ }^{a, c, d}$ \\ ${ }^{\mathrm{a} B I N D}$ - Behavioral Imaging and Neural Dynamics Center, and Departments of ${ }^{\mathrm{b}}$ Human Movement Science and \\ ${ }^{\mathrm{C}}$ Neuroscience and Imaging, University of Chieti-Pescara, Chieti, and d Casa di Cura Privata Villa Serena, Città S. \\ Angelo, Pescara, Italy; ${ }^{\mathrm{e}}$ Department of Neurology and BRaIN Center, University of New Mexico, ${ }^{\mathrm{f}}$ The Mind Research \\ Network, and ${ }^{9}$ Department of Pediatrics, University of New Mexico Health Sciences Center, Albuquerque, N. Mex., \\ and hepartment of Neurology, Children's Hospital Boston, Harvard Medical School, Boston, Mass., USA
}

\section{Key Words}

Spontaneous cortical oscillation · Human brain

development - Maturation of cortical rhythm • Prehension •

Pediatric magnetoencephalography

\begin{abstract}
Mu rhythm is an idling rhythm that originates in the sensorimotor cortex during rest. The frequency of mu rhythm, which is well established in adults, is $8-12 \mathrm{~Hz}$, whereas the limited results available from children suggest a frequency as low as $5.4 \mathrm{~Hz}$ at 6 months of age, which gradually increases to the adult value. Understanding the normal development of mu rhythm has important theoretical and clinical implications since we still know very little about this signal in infants and how it develops with age. We measured mu rhythm over the left hemisphere using a pediatric magnetoencephalography (MEG) system in 25 infants (11-47 weeks), 18 preschool children (2-5 years) and 6 adults (20-39 years) for two 5-min sessions during two intermixed conditions: a rest condition in which the hands were at rest, and a prehension condition in which the subject squeezed a pipette with
\end{abstract}

his/her right hand. In all participants, mu rhythm was present over the frontoparietal area during the rest condition, but was clearly suppressed during the prehension condition. Mu rhythm peak frequency, determined from the amplitude spectra, increased rapidly as a function of age from $2.75 \mathrm{~Hz}$ at 11 weeks to $8.25 \mathrm{~Hz}$ at 47 weeks $\left(r^{2}=0.83\right)$. It increased very slowly during the preschool period $(3.1 \pm 0.9$ years; $8.5 \pm$ $0.54 \mathrm{~Hz}$ ). The frequency in these children was, however, lower than in adults $(10.3 \pm 1.2 \mathrm{~Hz})$. Our results show a rapid maturation in spontaneous mu rhythm during the first year of life.

Copyright $\odot 2011$ S. Karger AG, Basel

\section{Introduction}

Recent neurophysiological and functional neuroimaging studies in adults have demonstrated the existence of a neuronal mechanism that matches perception and action, and permits action recognition and understanding [1-5]. This mechanism manifests as a cortical oscillation in the sensorimotor area that is strongly inhibited

\begin{tabular}{ll}
\hline KARGER & ( 2011 S. Karger AG, Basel \\
Fax +41613061234 & \\
$\begin{array}{l}\text { E-Mail karger@karger.ch } \\
\text { www.karger.com }\end{array}$ & $\begin{array}{l}\text { Accessible online at: } \\
\text { www.karger.com/dne }\end{array}$
\end{tabular}

Marika Berchicci

BIND - Behavioral Imaging and Neural Dynamics Center

Via dei Vestini, 33

IT-66100 Chieti Scalo (Italy)

Tel. +39 0871355 4221, E-Mail m.berchicci@gmail.com 
during the execution of an overt movement, such as prehension [6-8], as well as during observation [9-13] or imagination of a goal-directed action [14, 15].

In 1954, Gastaut and Bert [16] observed that a strong EEG oscillation was elicited by the sensorimotor cortex during rest and that this oscillation was blocked whenever the participant watching the boxing match identified himself with one of the active subjects projected on the screen. Gastaut and Bert termed this sensorimotor rhythm as 'the rythme en arceau' because of its waveform. This oscillatory phenomenon was initially named the Rolandic or central alpha rhythm in the EEG literature, because it was associated with sensorimotor cortices surrounding the central sulcus and had the same frequency range as the alpha rhythm. This oscillation, now called mu rhythm, is different from the alpha rhythm because the alpha rhythm originates in the occipital visual network and is attenuated when the eyes are open, whereas the mu rhythm reflects sensorimotor processing in the frontoparietal network [17] and is not significantly affected by opening or closing the eyes [18]. Mu rhythm did not receive the attention that other EEG oscillations have received because it was observed sporadically. Investigators have focused only recently on the neurophysiological basis of the rhythms associated with motor behavior and its observations in adults $[19,20]$.

A small number of studies have focused on mu rhythm and its evolution in infants and children. Smith [21] observed rhythmic 7-Hz EEG activity in children 4 months of age that originated from the central cortex. Using a cross-sectional sample, Smith [22] further observed that the mean frequency of this oscillatory activity slowly increased toward $8 \mathrm{~Hz}$ at 18 months of age, reached $9 \mathrm{~Hz}$ at about 4 years of age and finally stabilized around $10 \mathrm{~Hz}$ during adolescence. He interpreted the developmental onset of this central EEG activity during wakefulness as the loss of primitive reflexes and the appearance of voluntary control of neuromuscular behavior. Hagne [23] confirmed Smith's results in a longitudinal study of infants 6-12 months of age. They found that the central alpha rhythm was around $6 \mathrm{~Hz}$ at 6 months and that it increased to just above $7 \mathrm{~Hz}$ at 12 months. Both Smith and Hagne speculated on the contribution of developing motor and locomotor skills to the central alpha rhythm in infants. Some decades later, longitudinal studies provided the first evidence of a central rhythm in the alpha band that was suppressed during action performed by infants and was associated with adult sensorimotor mu rhythm $[24,25]$. In these studies, an age-related increase in the peak frequency of this alpha range central rhythm from 5 months (around $7 \mathrm{~Hz}$ ) to 4 years of age (around $9 \mathrm{~Hz}$ ) provided additional confirmation of the central alpha rhythm.

Fecteau et al. [26] reported modulation of EEG signals recorded over the sensorimotor area in a 36-month-old child undergoing intracranial surgery for intractable epilepsy. They observed an attenuation of the spectral power in the mu rhythm frequency band while the child was drawing with her right hand versus when she was watching an experimenter performing a similar movement. Fecteau et al. interpreted this mu rhythm attenuation as a reflection of sensorimotor processing in the frontoparietal network.

In order to investigate the effect of motor experience on motor resonance during observation, van Elk et al. [27] measured the spectral power in both mu and beta frequency bands in EEG signals recorded in twelve 14- to 16-month-old toddlers. They found a stronger motor resonance for the observation of actions that were already present in the infants' motor repertoire, and stronger mu and beta desynchronizations in relation to the infant's natural crawling experience. Nystrom [12] first showed that the observation of others' goal-directed actions is identifiable by mu rhythm modulations in infants as young as 6 months of age [12].

Although these studies have examined the characteristics of mu rhythm in infants and children, the development of this rhythm during the first months of life is still poorly characterized. Therefore, we have examined the evolution of mu rhythm during the first year of life and related this maturation all the way to adulthood. Our hypothesis was that mu rhythm can be observed during infancy and that mu rhythm peak frequency increases with chronological age. In order to test our hypothesis, we measured mu rhythm over the left hemisphere in typically developing infants, children and adults during rest and prehension using a pediatric MEG system (BabySQUID [28]).

\section{Materials and Methods}

\section{Subjects}

Data collection was performed in infants, children and adults using a cross-sectional design. Prior to participant recruitment, the protocol was reviewed and approved by the Human Research Review Committee at the University of New Mexico Health Sciences Center, Albuquerque, N. Mex., USA. Written informed consent was obtained from participants' parents or adult participants after the description of the study protocol. During the informed consent discussion, questions were invited and answered. Infants and children were recruited from the community using word of 
mouth, brochures posted on the campus, in the community and at day-care centers. Social networks were also used to recruit potential participants. Parents whose infants had participated in previous studies and had agreed to be contacted for future studies were contacted for recruitment purposes.

Infants and children were included if they were younger than 60 months of age. Infants and children were excluded from further participation if they had experienced any serious illnesses or developmental problems since birth (i.e. traumatic brain injury, seizures and congenital conditions), or if they were receiving any long-term medication.

Out of the 43 healthy infants enrolled ( $<12$ months of age), 25 infants met all inclusion and exclusion criteria, and cooperated during magnetoencephalographic data acquisition. The infants of our sample were born between 37 and 42 weeks of gestation (mean $=39.5, \mathrm{SD}=1.8$ ) and weighed between 2,500 and $3,800 \mathrm{~g}$ (mean $=3,000, \mathrm{SD}=0.600)$. Chronological age at entry to the study ranged between 11 and 47 weeks (mean $=26.6, \mathrm{SD}=11.9$ ). The functional development of all infants was examined with the Kent Inventory of Developmental Skills (KIDS [29]). All infants were found to function within the normal range for age. Parent report was used to assess Apgar scores [30] at birth, which were all within normal limits (i.e. 8-10).

An older group of 18 healthy children aged between 24 and 60 months (mean $=37.5, \mathrm{SD}=10.8)$ was enrolled and met all inclusion and exclusion criteria. According to parent report, none had known neurological abnormalities (seizures or other neurological diagnosis) or were on any long-term medication.

Six right-handed adults participated in the study to validate the method and served as a control group. They were recruited from the community using word of mouth and brochures posted on the campus or at the Mind Research Network in Albuquerque, N. Mex., USA. Participant age ranged from 20 to 39 years (mean $=28.3, \mathrm{SD}=7.8$ ), and no participant had a prior history of neurological illness.

\section{Experimental Setup}

Neuromagnetic activity was recorded by means of a magnetoencephalography (MEG) system, the BabySQUID, specifically developed for assessing brain function in infants [28]. Multichannel MEG systems allow completely noninvasive measurement of brain function and provide excellent temporal resolution, and, depending on the number of available sensors, good spatial resolution. A technical and practical advantage of using MEG systems to monitor brain activity in infants and children is that MEG signals are unaffected by immature skull features, such as fontanels, allowing for longitudinal neurodevelopmental studies.

The sensor array of the BabySQUID operates in a magnetically shielded room, and consists of 76 first-order axial gradiometers, with a pickup coil of $6 \mathrm{~mm}$ diameter and $30 \mathrm{~mm}$ baseline. Intersensor distance is about $13 \mathrm{~mm}$ from center to center. The pickup coils are placed $6 \pm 1 \mathrm{~mm}$ below the outer surface of the headrest, which is made of $0.75 \mathrm{~mm}$ thick fiberglass whose outer surface is smooth. The headrest was based on a standard reference for the head size of babies and has an ellipsoidal shape with a radius of curvature of $7.5 \mathrm{~cm}$ along the coronal section and $10 \mathrm{~cm}$ along the sagittal section. In neonates, the thickness of scalp and skull is about 3-4 $\mathrm{mm}$; therefore, brain activity can be measured a few millimeters above the brain surface. Given that the neuromagnetic field strength decreases with the squared distance be- tween source and sensor, the very short distance between the sensors and the brain sources provides excellent sensitivity. Furthermore, the high-density sensor array provides also high spatial resolution.

The BabySQUID system is equipped with a commercially available head-positioning system, visible with an infrared camera (IR Polaris camera). This system allows for measurement of head position before/during/after the scan to identify head position relative to the sensor array with high precision. This approach is similar to that employed by other commercially available MEG systems. However, this system requires compliance of the child with no movement during the head-positioning stage, and, in some cases, information on the shape of the baby's head and on its position with respect to the BabySQUID sensor array could not be collected. In spite of these challenges, we found the video recording, obtained in all children, to provide sufficient information for the purpose of this study for the following reasons. First, the head position was monitored via video recording during the entire duration of the MEG data acquisition unlike the snapshot of head position information provided by the Polaris camera. Second, it provided critical integrated information about stable head position in conjunction with task compliance. Although video recording does not provide the precision of the Polaris camera, it provides more than sufficient information about proper head positioning to ensure coverage of the sensorimotor area for the purpose of our study, based on the size of the sensor array. Further, the correctness of head positioning on the headrest was confirmed off line when the MEG signals, showing mu rhythm elicited by the Rolandic cortex, were identified by means of the functional topography approach (see next two sections).

\section{Experimental Design}

In order to perform prehension, infants, children and adult participants were directed to squeeze a pipette connected to a pressure transducer that continuously recorded the pressure exerted during prehension. The pressure profile was then sent to the BabySQUID electronics, so that the pressure waveform was synchronized with MEG data acquisition, permitting the investigator to identify the time points at which squeezing started. This was necessary for MEG data postprocessing purposes. The pipette was small enough to be comfortably held and squeezed by infants and children. The adults used a similar device with a button trigger similarly synchronized with the MEG data.

The experimental sessions were also recorded with a video camera in order to observe behavior and to record any movement that might have occurred during acquisitions. The video signal was synchronized with MEG. Although videos do not have the same temporal resolution as MEG data, spurious movements of the baby's head can be well documented, and the corresponding segments in MEG recordings can be considered as noisy data and disregarded.

The acquisition sessions were scheduled to suit the infants' and children's circadian rhythm, especially for the youngest infants. Parents were asked to schedule the appointments during the morning, and to bring their child awake, preferably right after naptime. Ideally, infants and young children should be cooperative and calm in order to obtain good quality MEG data, with no or minimal head movements. 
Children were positioned on the MEG bed on their left side, with their back supported by a pillow. The child's left hemisphere was then positioned on the BabySQUID headrest. If the child felt uncomfortable from lying still, or was getting drowsy and needed a break, data collection was stopped. In order to ensure child safety and to promote calmness during the acquisition, a parent attended to the child inside the magnetically shielded room. When the adult participants were measured, their left hemisphere was positioned on the BabySQUID headrest and the same procedures as for the infants and children were followed.

Before acquisition, the experimenter stood in front of the infant/child at a distance of approximately $40 \mathrm{~cm}$, and interacted with him/her in order to make the infant/child feel comfortable with the experimental environment and to motivate him/her to participate in the upcoming task. Once the infant/child was settled and completely still, MEG acquisition started and two 5-min blocks of continuous MEG data were recorded. The shielded room light was kept dim during all acquisition sessions in order to minimize distractions.

During MEG data collection, two different repeated experimental conditions were intermixed: rest and prehension conditions. Under the rest condition, the child remained motionless for about $10 \mathrm{~s}$ while the investigator stood in front of him/her and engaged the child visually to prevent any slight alpha rhythm modifications related to opening and closing the eyes. During the prehension condition, the experimenter offered the pipette to the child, who reached and squeezed it with his/her right hand. In the case of very young infants, the experimenter simply placed the pipette at about $5 \mathrm{~cm}$ from his/her right hand, and waited for his/her voluntary grasping movement, which is known to be already present in fetuses [31]. For children aged 24-60 months, or when the participant was an adult, the investigator simply asked the subject to voluntarily squeeze the pipette every $10 \mathrm{~s}$.

\section{Data Acquisition and Processing}

MEG data were recorded at a sampling rate of $500 \mathrm{~Hz}$. Although data acquisition was halted in case of significant displacement of the child's head, there were residual small movements (e.g. chewing/sucking and arm movements) that resulted in MEG artifacts because of the magnetic fields generated by muscle activation and head movement. Those artifacts, as well as $60-\mathrm{Hz}$ noise originating from the power line, were removed during MEG data preprocessing.

MEG data with artifacts that could not be easily eliminated based on the above criteria were rejected on the basis of careful visual inspection of all channel traces and video recordings; only the remaining data were used for further analysis.

A procedure called functional topography approach was used to identify MEG signals showing mu rhythm elicited by the Rolandic cortex, and to select the cluster of channels for further analysis of mu rhythm $[12,24,32,33]$. Three criteria had to be satisfied: (1) frequency characteristics: signal desynchronization during movement, as compared to the rest condition, was observed within a given frequency band $(<15 \mathrm{~Hz})$; (2) spatial distribution over the cortex: signal desynchronization was detected in a cluster of at least six channels positioned near the Rolandic cortex, as identified on the basis of the topographic channel array and the subject's head dimension, shape and position with respect to the BabySQUID headrest, and (3) functional reactivity to specific condition: the previous two conditions occurred time-locked to the onset of prehension, as observed in the pressure profiles and/ or in the video recordings.

In general, the cluster of channels showing mu rhythm elicited by the Rolandic cortex was not the same in all subjects. This was due to interindividual anatomical differences and to small differences occurring in the position of the subjects' head on the headrest of the BabySQUID. Off-line analysis of the signals in the six channels of the cluster identified with this procedure was performed using custom-made software developed in the Matlab environment.

Time-frequency analysis was performed to identify the epochs of oscillatory changes associated with self-paced prehension over the sensorimotor cortex in the contralateral hemisphere (left hemisphere for right hand prehension). A Hamming window of 512 samples and 24\% overlap was used. This analysis was performed on data previously filtered using a bandbass forward-reverse Butterworth filter of the third order. On the basis of the time-frequency analysis outcome, we identified 4-second time windows where spontaneous mu rhythm and mu rhythm desynchronization related to prehension were detected. The epochs related to spontaneous mu rhythm were selected anywhere during periods at rest. On the other hand, the epochs associated with movement, during which mu rhythm desynchronization occurred, started $1 \mathrm{~s}$ before movement onset, in order to account for the anticipation of cortical activity with respect to movement onset. The latter was identified by means of the pressure transducer profile and/or the analysis of the video recording. Consequently, the prehension epochs were averaged across multiple squeeze events, increasing the likelihood that non-time-locked activities were suppressed and motor activity was emphasized.

To compare mu rhythm peak desynchronization across different ages, and based on the scarcity of studies on mu rhythm desynchronization in infants younger than 6 months of age, the entire study population was divided into 4 cohorts, as follows: cohort $1(\mathrm{n}=14)$ from 11 to 24 weeks of age (average = 17.9 weeks; $\mathrm{SD}=5.6$ weeks); cohort $2(\mathrm{n}=11)$ from 26 to 47 weeks of age (average $=37.6$ weeks; $\mathrm{SD}=7.7$ weeks); cohort $3(\mathrm{n}=18)$ from 2 to 5 years of age (average $=3.1$ years; $\mathrm{SD}=0.9$ years), and cohort 4 $(n=6)$ from 20 to 39 years of age (average $=28.3$ years; $S D=7.8$ years).

A total of about 20 normalized epochs for both rest and prehension conditions were used for analyses without differences across cohorts (Wilks $\lambda=0.915 ; \mathrm{F}_{(6,88)}=0.667, \mathrm{p}=0.677$ ). For each channel, the Fast Fourier transform was performed on the selected epochs for both the rest and prehension conditions. The amplitude spectra obtained for brain activity at rest and during prehension were averaged across epochs. Given that the duration of all the epochs related to prehension was $4 \mathrm{~s}$, spectral resolution was $0.25 \mathrm{~Hz}$. Finally, the amplitude spectra of the brain activity at rest and during prehension were averaged across the six channels of the selected cluster. The two resulting condition-specific averaged spectra were compared to detect mu rhythm peak frequency, which was identified as the lowest frequency component at which we could observe significant desynchronization in the spectrum associated with prehension, quantified by a percent amplitude reduction greater than $40 \%$ with respect to the corresponding peak of the spontaneous rhythm spectrum.

A preliminary analysis with the same low and high cut off frequencies $(0-15 \mathrm{~Hz})$ across cohorts revealed that no additional 
Fig. 1. Schematic of the sensor placements over the left hemisphere of a 3-year-old child, showing the full sensor coverage of the area of interest. The six cluster channels, clearly positioned near the Rolandic cortex, are highlighted in light green (colors refer to online version) and numbered. Examples of the MEG signals recorded by the six cluster channels in the rest condition (1 s duration centered on a burst of mu rhythm activity) are shown on the right. Consistency of the signals recorded by the cluster channels may be observed. A vertical red line encompassing all signals enables the observation of synchronicity across signals. Small differences in signal amplitude and waveform are due to the varying distances and orientations of the sensors with respect to the signal source.

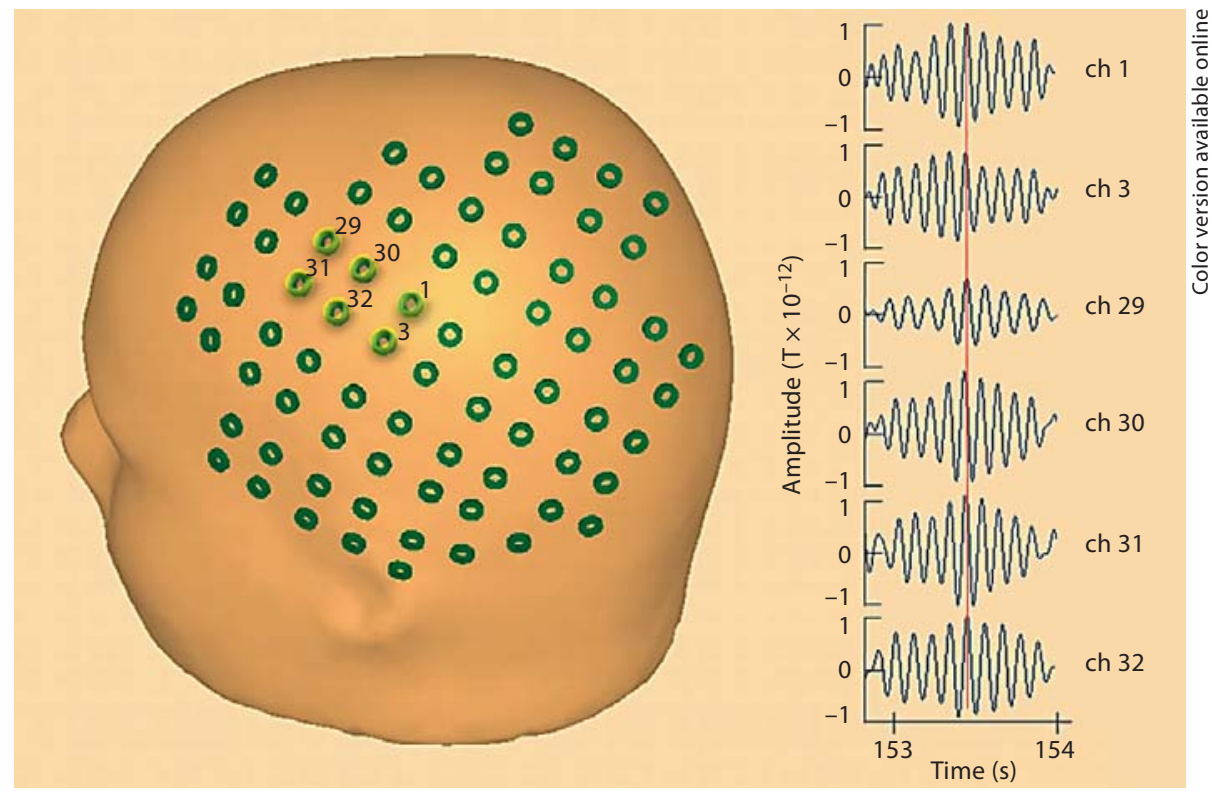

movement-related activity was present above $10 \mathrm{~Hz}$ in cohorts 1 and 2 and below $5 \mathrm{~Hz}$ in cohorts 3 and 4 . Moreover, recent research in infants and children has demonstrated that no adult model can be directly applied to children. We therefore adjusted the frequency bands according to the subject's age, and used a 0 to $10-\mathrm{Hz}$ band across infancy (11-47 weeks of age) and a 5- to 15$\mathrm{Hz}$ band across pediatric and adult groups, also according to findings from other examinations that suggest the application of ageadjusted frequency bands in developmental EEG and MEG research studies $[12,25,32]$. The described signal processing was performed in each participant. In order to test the statistical significance of the mu rhythm desynchronization detected as compared with the condition-specific averaged spectra for each cohort, we performed the following analysis. In each participant, we identified the mu rhythm peak frequency in the spectrum related to the rest condition, and selected three adjacent frequency windows of $1.5 \mathrm{~Hz}$ width, corresponding to 7 frequency bins: one window centered around mu rhythm peak frequency, one below and one above this central window. We identified the same frequency windows in the spectrum related to the prehension condition, and calculated the average amplitude for each window in the two spectra. We then performed a paired t test between the rest and prehension conditions for each cohort, using a Bonferroni multiple-comparisons correction to determine the $\mathrm{p}$ value for significance. We analyzed only three adjacent frequency windows of $1.5 \mathrm{~Hz}$ width instead of the entire spectrum because we did not want to include any beta band component in our analysis, and we know that these components may be close to the first harmonic of mu rhythm peak frequency [27].

We finally performed a paired $t$ test and a one-way ANOVA to highlight significant differences between conditions and groups.

\section{Results}

Figure 1 shows a schematic of the sensor placements over the left hemisphere of a 3-year-old child (sensor location was loaded into and displayed relative to the reference head surface using the commercially available MEG/ EEG analysis software BESA), showing full sensor coverage of the area of interest. The six cluster channels, selected on the basis of the functional topography approach, are highlighted in light green and numbered. They are clearly positioned near the Rolandic cortex. On the right, we show the MEG signals recorded by the six cluster channels under resting conditions (1 s duration centered on a burst of mu rhythm activity). Consistency within the signals recorded by the cluster channels may be observed, with small differences in signal amplitude and waveform due to the varying distances and orientations of the sensors with respect to the signal source.

Figure 2 illustrates the approach used to identify the cluster of contiguous channels positioned over the frontoparietal areas showing mu rhythm desynchronization in association with prehension. This example refers to a 14-week-old infant, and the two panels refer to a period during which prehension occurred (fig. $2 \mathrm{a}$ ) and to a period at rest (fig. 2b). In both panels, the length of the time window is $10 \mathrm{~s}$, and, from top to bottom, the panels show: (1) the MEG signal in a channel selected from the channel cluster (channel 25); (2) the spectrogram of the signal recorded at the selected channel, and (3) the simultaneous 


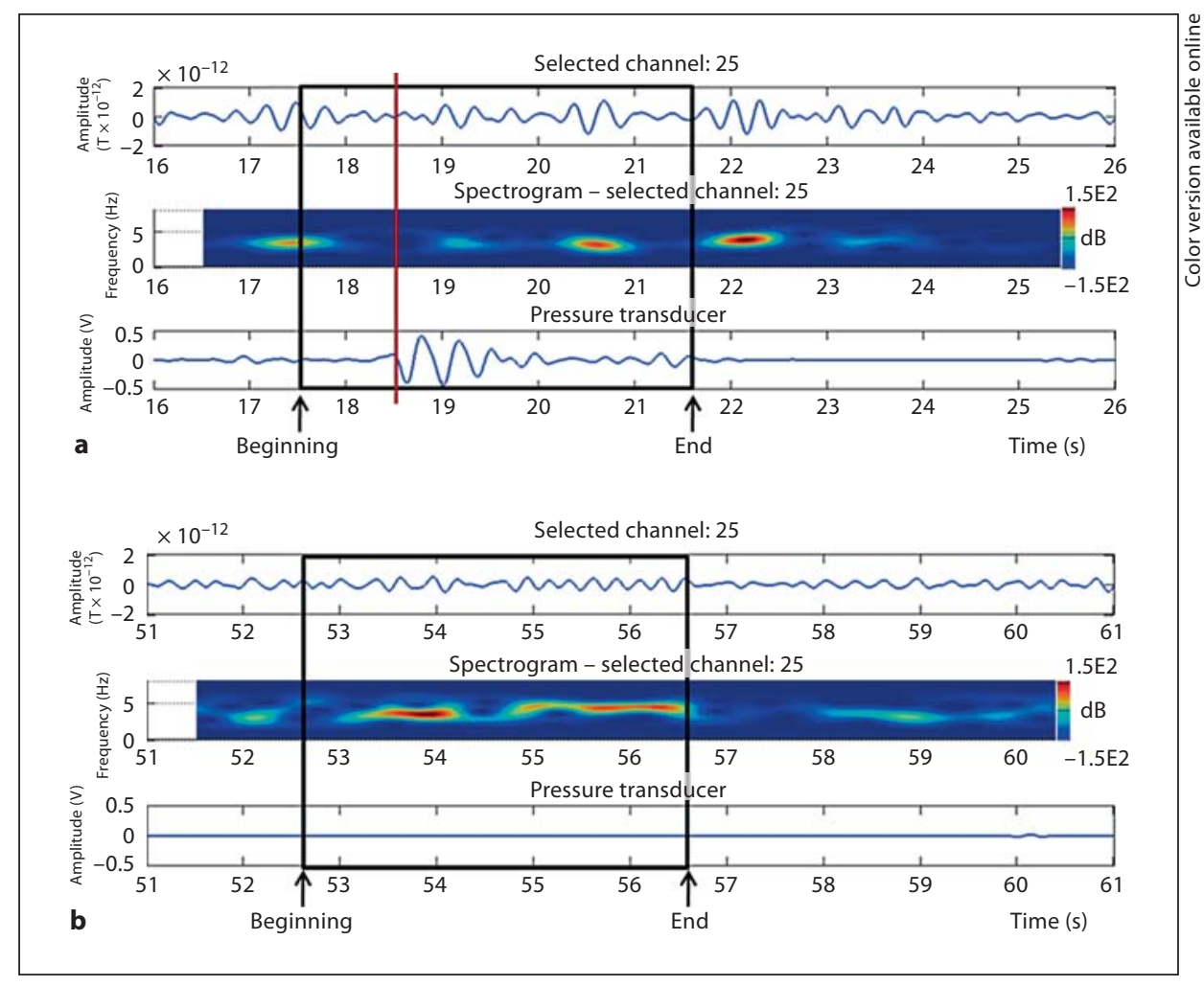

Fig. 2. Examples of temporal and time-frequency analysis performed on a 14-week-old infant over a period including prehension (a) and a period at rest (b). The information displayed from top to bottom in each panel is: MEG signal (in picoteslas) recorded at a selected channel near the Rolandic cortex (cluster channel 25); spectrogram showing the power spectrum of the signal in the selected channel as a function of time (frequency in hertz on the left $\mathrm{Y}$ axis, time from the beginning of acquisition in seconds on the $\mathrm{X}$ axis, spectral power attenuation in decibels $(\mathrm{dB})$ on the right $\mathrm{Y}$ axis); simultaneous pressure transducer profile (in volts) as a function of time. a Prehension onset is indicated by a vertical red line (color refers to online version); the 4-second time window used for the analysis of the prehension period is shown as a black square, and its beginning and end are indicated by arrows. It is worth noting that the epoch used to analyze prehension starts $1 \mathrm{~s}$ before movement onset. $\mathbf{b}$ The 4 -second time window used for the analysis of a period at rest is shown as a black square, and its beginning and end are indicated by arrows. At rest, epochs are selected anywhere during interprehension periods that have a minimum duration of $10 \mathrm{~s}$. pressure transducer profile, associated with prehension. The spectrogram is an estimate of the instantaneous spectral power in the analyzed signal. In all participants, time-frequency and waveform analysis of the MEG signals recorded over the sensorimotor areas demonstrated patterns of oscillatory activity in the mu rhythm frequency band, including: (1) the presence of mu rhythm prior to and after the execution of the motor task, and (2) mu rhythm suppression just before and during prehension. These patterns were consistent across all participants.

Comparison of the two condition-specific averaged spectra within participant (one related to brain activity at rest and the other related to brain activity during prehension) permitted identification of mu rhythm peak frequency for each participant. An example of the averaged spectra for each cohort is given in figure 3. The spectra show a relatively sharp, clear peak in amplitude during a rest period that diminished in the prehension condition. The peak frequency clearly increases with age. We performed a paired t test of the amplitude at the mu rhythm peak frequency obtained in all participants between rest and prehension conditions, and the amplitude differences were statistically significant $(p<0.01)$. In addition, the paired $t$ tests between the rest and prehension condition performed on the average amplitude values in the $1.5-\mathrm{Hz}$ windows centered around the mu peak for each cohort showed a significant condition-related difference $(\mathrm{p}<$ 0.001 ), whereas no statistically significant difference was observed between rest and prehension for the $1.5-\mathrm{Hz}$ frequency windows below $(p>0.1)$ and above $(p>0.1)$ the 

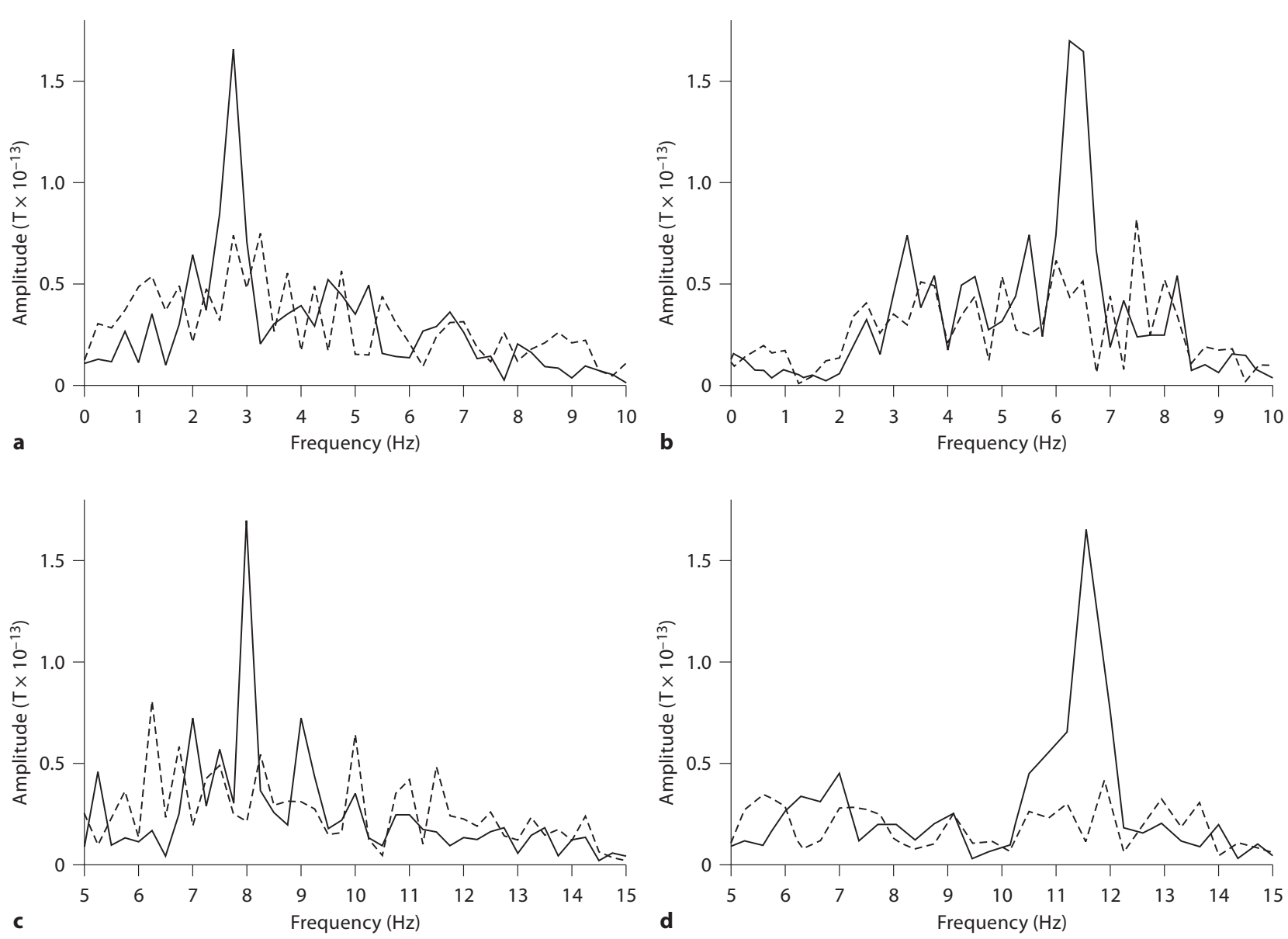

Fig. 3. Examples of averaged spectra for rest (—_) and prehension (- - ) conditions in 4 participants from cohorts $1-4$. Spectral resolution is $0.25 \mathrm{~Hz}$ because epochs of $4 \mathrm{~s}$ were considered. The spectra shown here are averaged across epochs for each channel and then averaged across the channels of the selected cluster above the left hemisphere, i.e. contralateral to the right hand. The main peak of mu rhythm activity clearly increases with age. a Amplitude spectrum for an 11-week-old infant with mu rhythm peak frequency at $2.75 \mathrm{~Hz}$. b Amplitude spectrum for a 28 -weekold infant with mu rhythm peak at $6.25 \mathrm{~Hz}$. c Amplitude spectrum for a 2-year-old child with mu rhythm peak at $8.00 \mathrm{~Hz}$. d Amplitude spectrum for a 28-year-old adult with mu rhythm peak at $11.50 \mathrm{~Hz}$. The frequency range in $\mathbf{a}$ and $\mathbf{b}$ corresponds to the bandpass used to analyze infant data $(0-10 \mathrm{~Hz})$ whereas the frequency range in $\mathbf{c}$ and $\mathbf{d}$ corresponds to the bandpass used to analyze child and adult data $(5-15 \mathrm{~Hz})$. mu rhythm peak frequency window (table 1). This result demonstrates that the detected frequency peaks are indeed related to mu rhythm since they show statistically significant reactivity to motor events whereas the activities in the surrounding frequency bands do not. We also verified the dependence of mu rhythm peak frequency on age. We detected a significant increase in mu rhythm peak frequency with age during the first year of life whereas no such increase was detected in older subjects (fig. 4). In infants from 11 to 47 weeks of age, we found a significant linear correlation between mu rhythm peak frequency and age (two-tailed Pearson correlation model, coefficient of determination $\mathrm{r}^{2}=0.83 ; \mathrm{p}<0.01$; calculated as the square of the sample correlation coefficient between the outcomes and their predicted values). After 2 years of age, individual mu rhythm peak frequencies are consistently higher than $7.50 \mathrm{~Hz}$. Please note that the total number of the dots visible in the lower panel is 23 instead of 25 (total number of subjects in cohorts 1 and 2) because there are two pairs of subjects at the same age and with 
Table 1. Repeated-measures $t$ test with Bonferroni correction between the rest and prehension conditions for each cohort

\begin{tabular}{|c|c|c|c|c|c|}
\hline \multirow[t]{2}{*}{ Cohorts } & \multirow[t]{2}{*}{ Frequency window } & \multicolumn{4}{|c|}{ Spectral average, $10^{-13} \mathrm{~T}$} \\
\hline & & rest condition & $\begin{array}{l}\text { prehension } \\
\text { condition }\end{array}$ & t-statistic & $\mathrm{p}$ value \\
\hline $\begin{array}{l}\text { Cohort } 1 \\
(\mathrm{n}=14)\end{array}$ & $\begin{array}{l}\text { below mu peak } \\
\text { centered at mu peak } \\
\text { above mu peak }\end{array}$ & $\begin{array}{l}0.93(0.53) \\
1.47(0.58) \\
0.75(0.31)\end{array}$ & $\begin{array}{l}0.86(0.47) \\
0.85(0.37) \\
0.70(0.23)\end{array}$ & $\begin{array}{l}1.75 \\
5.53 \\
1.43\end{array}$ & $\begin{array}{c}0.103 \\
<0.001^{*} \\
0.178\end{array}$ \\
\hline $\begin{array}{l}\text { Cohort } 2 \\
(\mathrm{n}=11)\end{array}$ & $\begin{array}{l}\text { below mu peak } \\
\text { centered at mu peak } \\
\text { above mu peak }\end{array}$ & $\begin{array}{l}1.28(0.45) \\
2.00(0.75) \\
0.99(0.58)\end{array}$ & $\begin{array}{l}1.24(0.41) \\
1.23(0.56) \\
0.95(0.59)\end{array}$ & $\begin{array}{l}0.63 \\
9.35 \\
0.88\end{array}$ & $\begin{array}{c}0.541 \\
<0.001^{*} \\
0.399\end{array}$ \\
\hline $\begin{array}{l}\text { Cohort } 3 \\
(\mathrm{n}=18)\end{array}$ & $\begin{array}{l}\text { below mu peak } \\
\text { centered at mu peak } \\
\text { above mu peak }\end{array}$ & $\begin{array}{l}0.14(0.08) \\
0.98(0.51) \\
0.88(0.50)\end{array}$ & $\begin{array}{l}0.14(0.10) \\
0.67(0.39) \\
0.84(0.43)\end{array}$ & $\begin{array}{r}-0.38 \\
5.44 \\
0.98\end{array}$ & $\begin{array}{c}0.709 \\
<0.001^{*} \\
0.343\end{array}$ \\
\hline $\begin{array}{l}\text { Cohort } 4 \\
(n=6)\end{array}$ & $\begin{array}{l}\text { below mu peak } \\
\text { centered at mu peak } \\
\text { above mu peak }\end{array}$ & $\begin{array}{l}0.41(0.16) \\
1.22(0.56) \\
0.56(0.18)\end{array}$ & $\begin{array}{l}0.40(0.13) \\
0.64(0.47) \\
0.51(0.26)\end{array}$ & $\begin{array}{l}0.11 \\
7.27 \\
0.67\end{array}$ & $\begin{array}{c}0.914 \\
<0.001^{*} \\
0.530\end{array}$ \\
\hline
\end{tabular}

The width of each frequency window is $1.5 \mathrm{~Hz}$, corresponding to 7 frequency bins in our spectra. The frequency windows below, around and above mu rhythm peak frequency are adjacent. The $t$ statistic and related $\mathrm{p}$ values are shown for each frequency window in the two right-hand columns. ${ }^{*} \mathrm{p}<0.004$ is significant with Bonferroni correction. Figures in parentheses are SDs.

the same mu rhythm peak frequency (see table A in suppl. material, www.karger.com/doi/10.1159/000329095).

It is also worth noting that the percent reduction in $\mathrm{mu}$ rhythm amplitude in the spectrum associated with prehension was greater than $55 \%$ in all participants, except for $2 / 49$ cases ( 1 infant and 1 child), in whom the spectrum amplitude reduction was around $40 \%$ (fig. 5). There was no obvious trend toward an increase or decrease in percent reduction even for the youngest age range (infants below 1 year).

Descriptive statistics of mu rhythm peak frequency by cohorts (table 2) show that the largest frequency range is observed in very early infancy (cohort 1 , age $\leq 24$ weeks, mu rhythm peak frequency between 2.75 and $6.0 \mathrm{~Hz}$ ). Mu rhythm during later infancy (cohort 2, age range 26-47 weeks) and childhood (cohort 3 , age range $2-5$ years) is still developing but with smaller increases in peak frequency (mu rhythm peak frequency between 6.3 and 9.5 $\mathrm{Hz}$ ), and their mu rhythm did not reach the same frequency values as in the adults in cohort 4 .

To examine our hypothesis of an increase in mu rhythm peak frequency with age, and to assess whether significant differences could be detected among cohorts, we performed a one-way ANOVA across the four cohorts (table 2). Findings reveal significant differences in mu rhythm peak frequency between groups $\left(\mathrm{F}_{(3,48)}=87.47\right.$, $\mathrm{p}<0.001$ ). Post hoc comparisons (Bonferroni correction) show that both cohort 1 and cohort 4 are significantly different from cohort 2 and cohort $3(\mathrm{p}<0.001)$, whereas no significant differences are observed between cohort 2 and cohort 3 . These results are consistent with the significant correlation with age shown for cohorts 1 and 2, demonstrating general increases in peak mu rhythm frequency with age during the first year of life.

\section{Discussion}

The primary contributions of this study were the detection of the motor-related suppression of mu rhythm in infants and the identification of the progressive increase in mu rhythm peak frequency from infancy to adulthood.

Despite the difficulties in recording functional data in young infants, the cross-sectional design of this study allowed the detection of mu rhythm activity in infants and young children. We observed that mu rhythm peak frequency linearly increases with age from 2.75 to $6.0 \mathrm{~Hz}$ in 


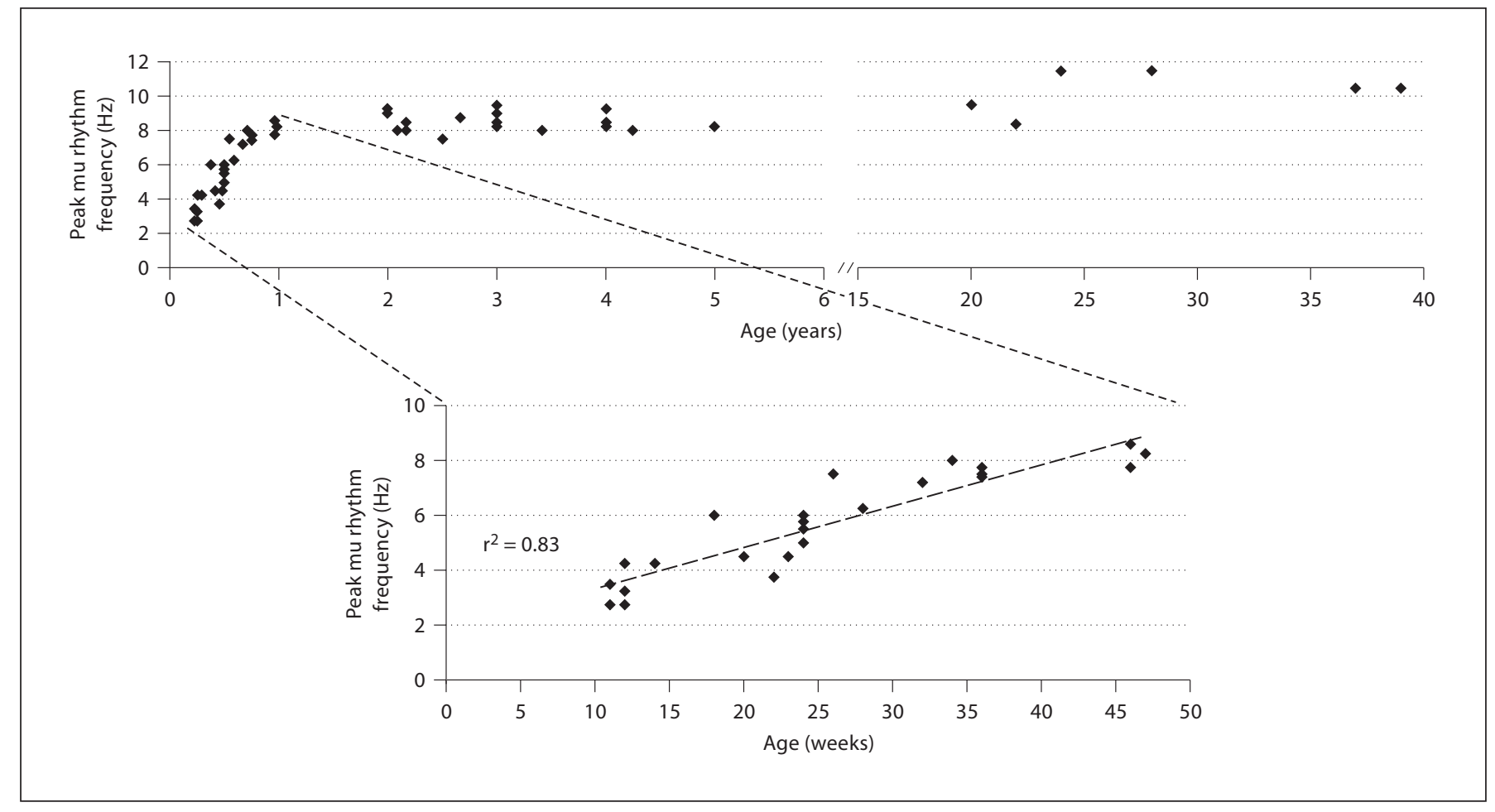

Fig. 4. Scatter plot of mu rhythm peak frequency as a function of age. The upper panel includes all participants whereas the lower panel shows the values obtained for the infants (cohorts 1 and 2). The high correlation between mu rhythm peak frequency and age $\left(r^{2}=0.83\right)$ in infants between 1 and 12 months of age is clearly visible from the plot in the lower panel.

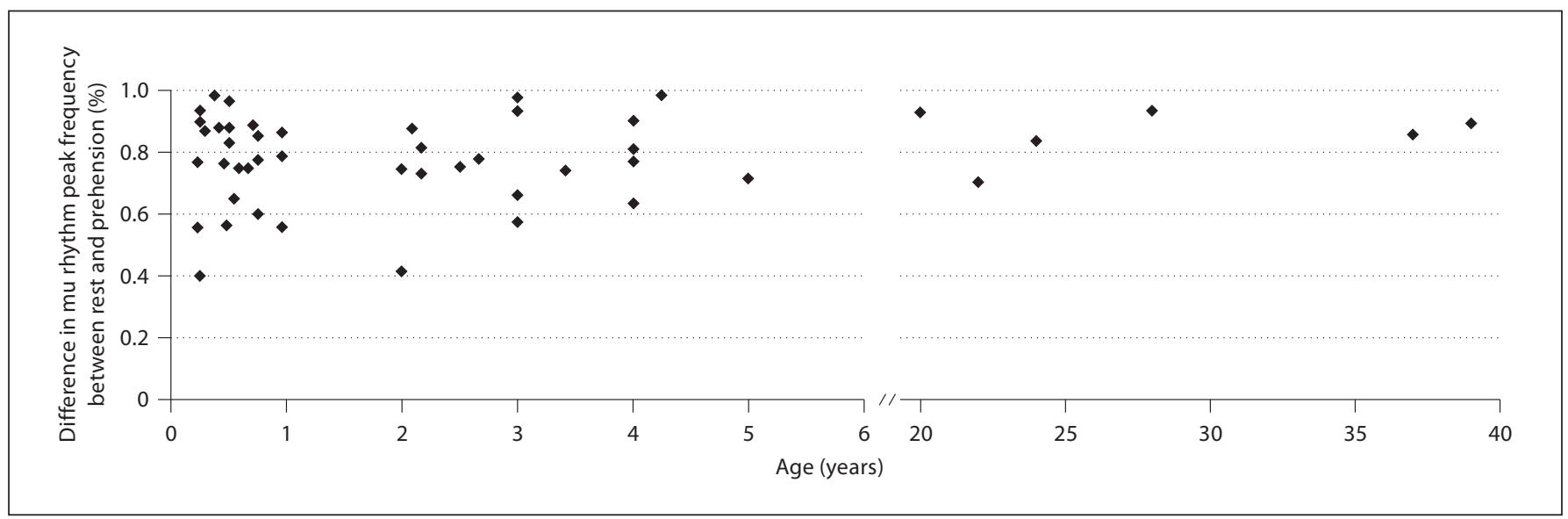

Fig. 5. Scatter plot of the percent difference in individual mu rhythm peak frequency amplitude between rest and prehension conditions as a function of age in all participants. No trend is visible, but it is notable that the percent amplitude reduction at mu rhythm peak frequency is always greater than $55 \%$ at all ages, except for 1 infant and 1 child. 
Table 2. Descriptive statistics for mu rhythm peak frequency and mu peak amplitude reduction from the rest to the prehension condition by cohorts

\begin{tabular}{|c|c|c|c|c|c|c|c|c|}
\hline \multirow[t]{2}{*}{ Cohorts } & \multicolumn{3}{|c|}{$\mathrm{Mu}$ peak frequency, $\mathrm{Hz}$} & \multirow{2}{*}{$\begin{array}{l}\text { Mu peak amplitude } \\
\text { reduction } \\
\text { mean } \pm \text { SD, } \%\end{array}$} & \multicolumn{4}{|c|}{ Post hoc comparison (Bonferroni) } \\
\hline & mean $\pm S D$ & $\min$ & $\max$ & & $\begin{array}{l}\text { groups } \\
\text { (a) }\end{array}$ & $\begin{array}{l}\text { groups } \\
\text { (b) }\end{array}$ & $\begin{array}{l}\text { mean } \\
\text { difference }(a-b)\end{array}$ & $\begin{array}{l}\mathrm{p} \\
\text { value }\end{array}$ \\
\hline $\begin{array}{l}\text { Cohort } 1 \\
(17.9 \pm 5.6 \text { weeks })\end{array}$ & $4.4 \pm 1.4$ & 2.7 & 6.0 & $0.80 \pm 0.17$ & 1 & $\begin{array}{l}2 \\
3 \\
4\end{array}$ & $\begin{array}{l}-3.4 \\
-4.3 \\
-6.1\end{array}$ & $\begin{array}{l}<0.001 \\
<0.001 \\
<0.001\end{array}$ \\
\hline $\begin{array}{l}\text { Cohort } 2 \\
(37.6 \pm 7.7 \text { weeks })\end{array}$ & $7.7 \pm 0.6$ & 6.3 & 8.6 & $0.75 \pm 0.10$ & 2 & $\begin{array}{l}1 \\
3 \\
4\end{array}$ & $\begin{array}{r}3.4 \\
-0.8 \\
-2.6\end{array}$ & $\begin{array}{r}<0.001 \\
0.144 \\
<0.001\end{array}$ \\
\hline $\begin{array}{l}\text { Cohort } 3 \\
(3.1 \pm 0.9 \text { years })\end{array}$ & $8.5 \pm 0.5$ & 7.5 & 9.5 & $0.77 \pm 0.14$ & 3 & $\begin{array}{l}1 \\
2 \\
4\end{array}$ & $\begin{array}{r}4.3 \\
0.8 \\
-1.8\end{array}$ & $\begin{array}{r}<0.001 \\
0.144 \\
<0.001\end{array}$ \\
\hline $\begin{array}{l}\text { Cohort } 4 \\
(28.3 \pm 7.8 \text { years })\end{array}$ & $10.3 \pm 1.2$ & 8.4 & 11.5 & $0.86 \pm 0.08$ & 4 & $\begin{array}{l}1 \\
2 \\
3\end{array}$ & $\begin{array}{l}6.1 \\
2.6 \\
1.8\end{array}$ & $\begin{array}{l}<0.001 \\
<0.001 \\
<0.001\end{array}$ \\
\hline
\end{tabular}

infants from 11 to 47 weeks of age, and that the peak frequency at 6 months is in agreement with previous data $[12,21-27,32,34]$. Despite the expected interindividual differences, we observed that the largest mu rhythm peak frequency change occurs during the first year of life (from $2.75 \mathrm{~Hz}$ at 11 weeks to $8.6 \mathrm{~Hz}$ at 47 weeks), and that this linear increase is statistically significant (coefficient of determination $\mathrm{r}^{2}=0.83$ ). The increase in mu rhythm peak frequency with age might be explained by the rapid development of the structural and functional underpinnings of cortical neural networks [35], by the changes in the physical orientation and density of neuronal assemblies, and by both synaptogenesis and the development of myelination across the cortex [36]. Each of these processes begins during gestation and continues after birth.

In our study, the time-frequency analysis, the MEG signal waveform analysis and the position of the cluster channels with respect to the participant's head demonstrate that the oscillatory activity observed in all participants is largest at rest, is damped shortly before movement onset, and is suppressed during prehension. These observations are in agreement with previous findings on the nature, cortical origin and dynamics of mu rhythm $[11,12,37]$ and suggest a sensorimotor generator site for this oscillatory activity. The reliability of our results is also confirmed by the high percentage of mu rhythm desynchronization during prehension. Indeed, we measured a percent desynchronization of mu rhythm at peak frequencies greater than $55 \%$ in all participants, except for one 3-month-old infant (40\%) and one 2-year-old child (40\%). This variability is consistent with early reports showing difficulty in identifying mu rhythm activity in all individuals [38]. The consistency across most individuals is in agreement with previous results suggesting that MEG is more sensitive to mu rhythm than EEG. The strong attenuation of mu rhythm spectral amplitude during voluntary prehension was also confirmed by the paired $t$ test performed for each cohort, and is in agreement with the well-known condition of maximum mu rhythm amplitude during rest and its decrease during goal-directed action $[39,40]$.

According to Orekhova et al. [41], who previously observed left-sided predominance of mu rhythm under all experimental conditions in children, we measured mu rhythm activity from the left hemisphere for each cohort. In fact, the infants reached for the pipette only with the right hand while they were lying on their left side. In contrast to Orekhova et al., we observed that the increase in mu rhythm peak frequency is characterized by an upward shift of the mu rhythm band toward higher fre- 
quencies from infancy $(2.75-8.6 \mathrm{~Hz})$ to adulthood $(8.4-$ $11.5 \mathrm{~Hz}$ ) rather than by an expansion of the mu rhythm band. It has also been suggested that theta activity may be the precursor of alpha and mu rhythms [24, 42]. The theta band in preschool children is close to the adult value $(4-8 \mathrm{~Hz})$ and broader than in infants $(4-6 \mathrm{~Hz})$. However, theta rhythm synchronizes under conditions of emotional and cognitive load $[41,43,44]$ with predominance over associative (parietal, temporal, or frontal) cortical areas and over the projection of the hippocampus. The differential activation of mu rhythm and theta activity is critical for selectively characterizing mu rhythm. Our experimental design allowed us to selectively average epochs related to rest or prehension, which will tend to average out theta activity related to emotional and cognitive processes. Moreover, our results on mu rhythm peak frequency in older infants (cohort 2, 26-47 weeks, $6.3-8.6 \mathrm{~Hz}$ ) are in accordance with previous findings on the frequency range of mu rhythm $(6.0-9.0 \mathrm{~Hz})$ in agematched infants $[12,24,25,34]$. Similarly, the results found within the adult cohort (cohort 4) are in agreement with previous findings that report a frequency range of $8-12 \mathrm{~Hz}$, and validate the methods of analysis used in this study $[3,4,45-47]$. Finally, the topography of our signals indicates that they are mu rather than theta rhythms. Therefore, although theta and mu frequency bands may overlap in infancy, we do not consider the activity identified in this study to be theta activity.

The statistical analysis across cohorts provides partial confirmation of our main hypothesis. We were able to observe mu rhythm during infancy and a significant difference in the distribution of mu rhythm peak frequency between the cohort of the youngest infants (cohort 1) and all other cohorts, suggesting that changes in mu rhythm peak frequency occur across specific chronological ages. Consistent with Niedermayer [38], our findings further indicate that mu rhythm peak frequency continues to develop after 5 years of age. Only sparse data on the development of mu rhythm in infancy and childhood are available [12, 21-27, 40, 42], and, to our knowledge, the current study provides the first clear evidence of systematic changes in mu rhythm during the first months of life.

Until recently, the research on early human sensorimotor development was dominated by Piaget's [48] view, which affirms that perception and action are dissociable processes that are coordinated later through experience. This theory implies that infants initially perform behaviors randomly. However, research has challenged this historical perspective and has provided evidence for the coupling of perception and action beginning at birth [49-51]
One of the most remarkable examples of perception and action coupling in infants is prehension since reaching for an object is guided by perceptual information regarding the relation between the self and the environment, which continuously changes as the posture (e.g. the motor system) is adjusted relative to that information [52]. According to Aslin [53], saccadic eye movements following the target are detectable by 6 weeks of age, after which, smooth pursuit starts to be observed. Smooth pursuit was clearly present at 2 months of age and the compensatory head movements were coordinated with the tracking eye at almost unity gain [54]. However, there are other examples of perception-action coupling and prospective goal-directed behaviors in infants [55-59] that show how infants seem to be sensitive to the goal structure of action with respect to the object or outcomes to which the action is directed. With some experience, infants become able to map their observation of an action onto preliminary motor representations, as revealed by a few studies employing a habituation task $[60,61]$. In agreement with those findings, Sommerville et al. [62] showed that infants as young as 3 months can rapidly form goal-based action representations, and suggest a developmental link between infants' goal-directed actions and their ability to detect goals in the actions of others. Further, Clifton et al. [63] observed that 14-week-old infants reach rapidly and accurately in the dark for sounds as well as for luminous objects, demonstrating that they are capable of performing actions that are regulated by perceptual information. Indeed, it is now recognized that infants can perform coordinated actions as soon as the necessary muscle synergies are available [64-66]. At a neuronal level, infants as young as 6 months are capable of internally generating novel movements performed by others by assembling primitive movements already present in their motor repertoire [34, 67, 68]. These brain phenomena occurring during both covert and overt behaviors are reflected in the modulation of mu rhythm over the sensorimotor cortex [46]. Thus, action perception and neural development may enable infants to continually improve their affordances in the environment $[52,67]$. Taken together, we can speculate that newborns enter the world prepared to perceptually regulate actions that are essential to survival and adaptation, wherein development involves a confluence of factors that include biomechanical changes as well as environmental and task factors in conjunction with neural changes.

The brain undergoes fast and substantial transformations during the first months of life [25], and our findings provide additional information on the functional chang- 
es occurring in the sensorimotor areas of the brain in infants and children. This information might reveal useful markers for special populations of infants. In a developmental context, impaired performance of skilled gestures, including imitation, may be secondary to abnormalities in the frontoparietal circuits dedicated to the acquisition of the sensory representations of movement and motor sequence programs necessary to execute them, which are reflected in mu rhythm modulation and are part of the mirror neuron system (MNS) [69]. In fact, adult functional neuroimaging studies demonstrated that both mu rhythm and the MNS are tuned to goaldirected actions rather than just movements [70-72], and that mu rhythm suppression can be used as a proxy measure of MNS functioning [73, 74]. The characteristics of the MNS indicate that it plays an important role in social functions, such as general communication, imitation learning, and action understanding [75-77]. This suggests that the MNS is likely to be crucial to these same processes in children.

Finally, future research will help to address the limitations of the current study. First, study protocols like ours are difficult to perform in children and even more so in infants, which resulted in a small sample size and a wide age range. Subsequent developmental research that longitudinally follows mu rhythm maturation during infancy would provide additional validation of the current findings and extend our understanding of the emergence and sequential development of mu rhythm through infancy and early childhood. However, as concerns the current study, several research studies in adults and children have been considered reliable even when based on a smaller number of subjects [11, 42, 45, 71,78]. Second, there were limitations concerning source localization.
Even though previous EEG studies with infants [12, 27, 42] identified the parietal lobe as a core region for perception-action coupling for its connections to both visual and frontal motor areas, more findings are needed to better identify the source of mu rhythm signals [79]. A more precise localization has both theoretical and clinical implications, and may be central to improving our understanding of normal infant brain development and perturbations that can occur. Further, one common concern in studies with infants is the small number of trials that can be recorded during the experimental session, which may potentially limit the interpretation and generalizability of the results. However, we included a total of about 20 normalized epochs for each condition to calculate the averaged amplitude spectra for each cohort, wherein at least 20 prehension epochs were used to calculate the averaged amplitude spectra for each channel in order to increase the likelihood of detecting only mu rhythm. Subsequent longitudinal studies specifically designed to address activity in alpha, theta and mu frequency bands could provide more insight into their specific features and replicate our results.

\section{Acknowledgements}

We thank the Mind Research Network for providing access to the VAC magnetically shielded room for the BabySQUID MEG measurements. We thank Laura Urrea for her help with data collection. We also thank Steffen Bollmann and Denner Guilhon for their assistance in data analysis. This work was supported in part by a grant from the University of New Mexico Clinical \& Translational Science Center Novel Methods Award, by the Progetto Operativo Regionale Abruzzo 2000-2006 C3/IC4E grant to M.B., and by NIH R21-NS057614 (Y.O.).

\section{References}

1 Hari R, Levanen S, Raij T: Timing of human cortical functions during cognition: role of MEG. Trends Cog Sci 2000;4:455-462.

$\checkmark 2$ Hummel F, Andres F, Altenmuller E, Dichgans J, Gerloff C: Inhibitory control of acquired motor programs in the human brain. Brain 2002;125:404-420.

-3 Muthukumaraswamy SD, Johnson BW: Changes in Rolandic mu rhythm during observation of a precision grip. Psychophysiology 2004;41:152-156.
4 Muthukumaraswamy SD, Johnson BW, McNair NA: Mu rhythm modulation during observation of an object-directed grasp. Brain Res Cogn Brain Res 2004;19:195-201.

- 5 Depretto M, Davies MS, Pfeifer JH, Scott AA, Sigman M, Bookheimer SY, Iacoboni M: Understanding emotions in others: mirror neuron dysfunction in children with autism spectrum disorders. Nat Neurosci 2006;9: $28-30$.

-6 Jeannerod M, Arbib MA, Rizzolatti G, Sakata H: Grasping objects: the cortical mechanisms of visuomotor transformation. Trends Neurosci 1995;18:314-320.
7 Hari R, Forss N, Avikainen S, Kirveskari E, Salenius S, Rizzolatti G: Activation of human primary motor cortex during action observation: a neuromagnetic study. Proc Natl Acad Sci USA 1998;95:15061-15065.

8 Castiello U: The neuroscience of grasping. Nat Rev Neurosci 2005;6:726-736.

-9 Decety J, Grezes J, Costes N, Perani D, Jeannerod M, Procyk E, Grassi F, Fazio F: Brain activity during observation of actions. Influence of action content and subject's strategy. Brain 1997;120:1763-1777. 
10 Jarvelainen J, Schurmann M, Hari R: Activation of the human primary motor cortex during observation of tool use. Neuroimage 2004;23:187-192.

-11 Nishitani N, Hari R: Temporal dynamics of cortical representation for action. Proc Natl Acad Sci USA 2000;97:913-918.

12 Nystrom P: The infant mirror neuron system studied with high density EEG. Soc Neurosci 2008;3:334-347.

13 Vogt S, Buccino G, Wohlschlager AM, Canessa N, Shah NJ, Zilles K, Eickhoff SB, Freund HJ, Rizzolatti G, Fink GR: Prefrontal involvement in imitation learning of hand actions: effects of practice and expertise. Neuroimage 2007;37:1371-1383.

-14 Grafton ST, Arbib MA, Fadiga L, Rizzolatti G: Localization of action representations in humans by positron emission tomography. 2. Observation compared with imagination. Exp Brain Res 1996;112:103-111.

15 Molnar-Szakacs I, Kaplan J, Greenfield PM, Iacobini M: Observing complex action sequences: the role of fronto-parietal mirror neuron system. Neuroimage 2006;33:923935.

16 Gastaut HJ, Bert J: EEG changes during cinematographic presentation. Electroencephalogr Clin Neurophysiol 1954;6:433-444

- 17 Pineda JA: The functional significance of mu rhythms: translating 'seeing' and 'hearing' into 'doing'. Brain Res Brain Res Rev 2005; 50:57-68.

18 Kuhlman WN: Functional topography of the human mu rhythm. Electroencephalogr Clin Neurophysiol 1978;44:83-93.

-19 Pfurtscheller G, Neuper C, Pichler-Zalaudek K, Edlinger G, Lopes da Silva FH: Do brain oscillations of different frequencies indicate interaction between cortical areas in humans? Neurosci Lett 2000;286:66-68.

-20 Fadiga L, Craighero L: Electrophysiology of action representation. J Clin Neurophysiol 2004:21:157-169.

21 Smith JR: The 'occipital' and 'pre-central' rhythms during the first two years. J Physiol 1939;7:223-226.

22 Smith JR: The frequency growth of the human alpha rhythms during normal infancy and childhood. J Physiol 1941;11:177-198.

23 Hagne I: Development of the EEG in normal infants during the first year of life. A longitudinal study. Acta Paediatr Suppl 1972;232: $1-53$.

-24 Stroganova TA, Orekhova EV, Posikera IN: EEG alpha rhythm in infants. Clin Neurophysiol 1999;110:997-1012.

-25 Marshall PJ, Bar-Haim Y, Fox NA: Development of the EEG from 5 months to 4 years of age. Clin Neurophysiol 2002;113:1199-1208.

26 Fecteau S, Carmant L, Tremblay C, Robert M, Bouthiller A, Theoret H: A motor resonance mechanism in children? Evidence from subdural electrodes in a 36-month-old child. NeuroReport 2004;15:2625-2627.
27 van Elk M, van Schie HT, Hunnius S, Vesper $\mathrm{C}$, Bekkering $\mathrm{H}$ : You'll never crawl alone: neuropsychological evidence for experiencedependent motor resonance in infancy. Neuroimage 2008;43:808-814.

28 Okada Y, et al: BabySQUID: a mobile, highresolution multichannel magnetoencephalography system for neonatal brain assessment. Rev Sci Instruments 2006;77:1-9.

29 Reuter J, Katoff L, Gruber C: Kent Inventory of Developmental Skills (KIDS). Los Angeles, Western Psychological Services, 1996.

30 Apgar V: A proposal for a new method of evaluation of the newborn infant. Anesth Analg 1953;32:260-267.

31 Zoia S, Blason L, D’Ottavio G, Bulgheroni M, Pezzetta E, Scabar A, Castiello U: Evidence of early development of action planning in the human fetus: a kinematic study. Exp Brain Res 2007;176:217-226.

32 Orekhova EV, Stroganova TA, Posikera IN, Elam M: EEG theta rhythm in infants and preschool children. Clin Neurophysiol 2006; 117:1047-1062.

33 Kuhlman WN: The mu rhythm: functional topography and neural origin, in Pfurtsheller G (ed): Rhythmic EEG Activities and Cortical Functioning. Amsterdam, Elsevier, 1980, pp 105-120.

34 Shimada S, Hiraki K: Infant's brain responses to live and televised action. Neuroimage 2006;32:930-939.

35 Thatcher RW: Cyclic cortical reorganization during early childhood. Brain Cogn 2004;28: 24-50.

36 Purves D: Neural Activity and The Growth of the Brain. Cambridge, Cambridge Univer sity Press, 1994.

37 Hari R, Salmelin R: Human cortical oscillations: a neuromagnetic view through the skull. Trends Neurosci 1997;20:44-49.

38 Niedermayer E: Alpha rhythm as physiological and abnormal phenomena. Int J Psychophysiol 1997;26:31-49.

39 Mulholland T: Human EEG, behavioral still ness, and biofeedback. Int J Psychophysiol 1995;19:263-279.

40 Southgate V, Johnson MH, Osborne T, Csibra G: Predictive motor activation during observation in human infants. Biol Lett 2009;5:769-772.

41 Orekhova EV, Stroganova TA, Posikera IN: Theta synchronization during sustained anticipatory attention in infants over the second half of the first year of life. Int J Psychophysiol 1999;32:151-172.

-42 Lapage JF, Theoret H: EEG evidence for the presence of an action observation-execution matching system in children. Eur J Neurosci 2006;23:2505-2510.

43 Aftanas LI, Reva NV, Varlamov AA, Pavlov SV, Makhnev VP: Analysis of evoked EEG synchronization and desynchronization in conditions of emotional activation in humans: temporal and topographic characteristics. Neurosci Behav Physiol 2004;34:859867.
44 Kahana MJ, Seelig D, Madsen JR: Theta returns. Curr Opin Neurobiol 2001;11:739744.

45 Simoes C, Salenius S, Curio G: Short-term prediction of perturbation dynamics for 10and $20-\mathrm{Hz}$ MEG rhythms in human primary sensorimotor hand cortices. Neuroimage 2004;22:387-393.

46 Gaetz W, Cheyne D: Localization of sensorimotor cortical rhythms induced by tactile stimulation using spatially filtered MEG. Neuroimage 2006;30:899-908.

47 Hari R: Action-perception connection and the cortical mu rhythm. Prog Brain Res 2006;159:253-260.

48 Piaget J: The Origins of Intelligence in Children. New York, International University Press, 1952.

49 Anisfeld M: Review: neonatal imitation. Dev Rev 1991;11:60-97.

50 Adolph K, Joh A: Motor development: how infants get into the act; in Slater A, Lewis M (eds): Introduction to Infant Development, ed 2. Oxford, University Press, 2006, pp 171207

51 Meltzoff AN, Moore MK: Imitation, memory, and the representation of persons. Infant Behav Dev 1994;17:83-100.

52 Hatwell Y: Motor and cognitive functions of the hand in infancy and childhood. Int J Behav Dev 1987;10:509-526.

53 Aslin R: Development of smooth pursuit in human infants; in Fischer DF, Monty RA, Senders EJ (eds): Eye Movements: Cognition and Vision Perception. Hillsdale, Erlbaum, 1981, pp 31-51.

54 Von Hofsten C, Rosander K: Development of smooth pursuit tracking in young infants. Vision Res 1997;37:1799-1810.

55 Zelazo PR, Brody LR, Chaikan H: Neonatal habituation and dishabituation of head turning to rattle sounds. Infant Behav Dev 1984; 7:311-321.

56 Blass EM, Fillion TJ, Rochat P, Hoffmeyer LB, Metzger MA: Hand-mouth coordination in neonates. Dev Psychol 1989;25:963-975.

57 Butterworth GE, Hopkins B: Hand-mouth coordination in the newborn baby. Br J Dev Psychol 1988;6:303-314.

58 Bertenthal BI: Origins and early development of perception, action, and representation. Annu Rev Psychol 1996;47:431-459.

59 Bertenthal BI: Perception and action, in Haith M, Benson J (ed): Encyclopedia of Infant and Early Childhood Development. Oxford, Elsevier, 2008.

60 Streri A, Féron J: The development of haptic abilities in very young infants: from perception to cognition. Infant Behav Dev 2005;28: 290-304.

61 Sann C, Streri A: Inter-manual transfer of object texture and shape in human neonates. Neuropsychologia 2008;46:698-703. 
-62 Sommerville JA, Woodward A, Needham A: Action experience alters 3-month-old infants' perception of others' actions. Cognition 2005;96:B1-B11.

63 Clifton RK, Muir DW, Ashmead DH, Clarkson MG: Is visually guided reaching in early infancy a myth? Child Dev 1993;64:10991110.

64 Sporns O, Edelman GM: Solving Bernstein's problem: a proposal for the development of coordinated movement by selection. Child Dev 1993;64:960-981.

65 Warren W: The perception-action coupling, in Bloch H, Bertenthal BI (eds): Sensory-Motor Organization and Development in Infancy and Early Childhood. Dordrecht, Kluwer, 1990, pp 23-38.

66 Von Hofsten C: Catching skills in infancy. J Exp Psychol Hum Percept Perform 1983;9: 75-85.

67 Thelen E, Corbetta D, Kamm K, Spencer JP, Schneider K, Zernicke RF: The transition to reaching: mapping intention and intrinsic dynamics. Child Dev 1993;64:1058-1098.
68 Newell KM: Constraints on the development of coordination; in Wade MG, Whiting HTA (eds): Motor Development in Children: Aspects of Coordination and Control. Boston, Martinus Nijhoff, 1986, pp 341-360.

69 Williams JHG, Whiten A, Singh T: A systematic review of action imitation in autistic spectrum disorder. J Autism Dev Disord 2004;34:285-299.

70 Rumiati RI, Weiss PH, Tessari A, Assmus A, Zilles K, Herzog H, Gereon RF: Common and differential neural mechanisms supporting imitation of meaningful and meaningless actions. J Cogn Neurosci 2005;17: 1420-1431.

71 van Schie HT, Koelewijn T, Jensen O, Oostenveld R, Maris E, Bekkering H: Evidence for fast, low-level motor resonance to action observation: an MEG study. Soc Neurosci 2008; 3:213-228.

72 Zarinah KA, Bhakoo KK, Basant KP: The human mirror system: a motor resonance theory of mind-reading. Brain Res Rev 2007; 54:286-293.

73 Oberman LM, Hubbard EM, McCleery JP, Altschuler EL, Ramachandran VS, Pineda JA: EEG evidence for mirror neuron dysfunction in autism spectrum disorders Cogn Brain Res 2005;24:190-198.
74 Rizzolatti G, Craighero L: The mirror neuron system. Annu Rev Neurosci 2004;27: 169-192.

75 Adolphs R: Cognitive neuroscience of human social behaviour. Nat Rev Neurosci 2003;4:165-178.

76 Fuji N, Hihara S, Hiriki A: Social cognition in premotor and parietal cortex. Soc Neurosci 2008;3:250-260.

77 Leslie KR, Johnson-Frey SH, Grafton ST: Functional imaging of face and hand imitation: towards a motor theory of empathy. Neuroimage 2004;21:601-607.

78 Salmelin R, Hamalainen M, Kajola M, Hari R: Functional segregation of movement-related rhythmic activity in the human brain. Neuroimage 1995;2:237-243.

79 Marshall PJ, Meltzoff AN: Neural mirroring system: exploring the EEG mu rhythm in human infancy. Dev Cogn Neurosci 2011;1: 110-123. 